\title{
Real World Bayesian Optimization Using Robots to Clean Liquid Spills
}

\author{
Isobel Voysey ${ }^{1}$, Josie Hughes ${ }^{1}$, Thomas George Thuruthel ${ }^{1}$, Kieran Gilday ${ }^{1}$, \\ and Fumiya Iida ${ }^{1}$ \\ Bio-Inspired Robotics Laboratory, Department of Engineering, the University of \\ Cambridge, UK \{iv256, jaeh2, tg444, kg398,fi224\}@cam.ac.uk
}

\begin{abstract}
Developing robots that can contribute to cleaning could have a significant impact on the lives of many. Cleaning wet liquid spills is a particularly challenging task for a robotic system, and has several high impact applications. This is a hard task to physically model due to the complex interactions between cleaning materials and the surface. As such, to the authors' knowledge there has been no prior work in this area. A new method for finding optimal control parameters for the cleaning of liquid spills is required by developing a robotic system which iteratively learns to clean through physical experimentation. The robot creates a liquid spill, cleans and assesses performance and uses Bayesian optimization to find the optimal control parameters for a given size of liquid spill. The automation process enabled the experiment to be repeated more than 400 times over 20 hours to find the optimal wiping control parameters for many different conditions. We then show that these solutions can be extrapolated for different spill conditions. The optimized control parameters showed reliable and accurate performances, which in some cases, outperformed humans at the same task.
\end{abstract}

Keywords: Robotic cleaning · Control Optimization · Service Robotics.

\section{Introduction}

In the coming years service robots are expected to have significant impacts on our daily lives [2]. The kitchen is one place where robotics has the potential to make a significant impact and to improve quality of life for many individuals [20]. One particular kitchen task which is widely disliked is cleaning, yet it is key for maintaining the cleanliness and food hygiene standards in a kitchen [4]. One of the most challenging cleaning tasks is cleaning wet surfaces. Such surfaces are also one of the most high risk zones of the kitchen with regards to the spread of unwanted disease [6]. Such robots could improve users quality of life and assist with enabling the elderly to live independently in their homes for longer [19]. In addition, the ability to clean liquid spills could enable robots to clear up hazardous liquid waste, assisting in tasks such as nuclear decommissioning or in high risk lab environments [17]. 


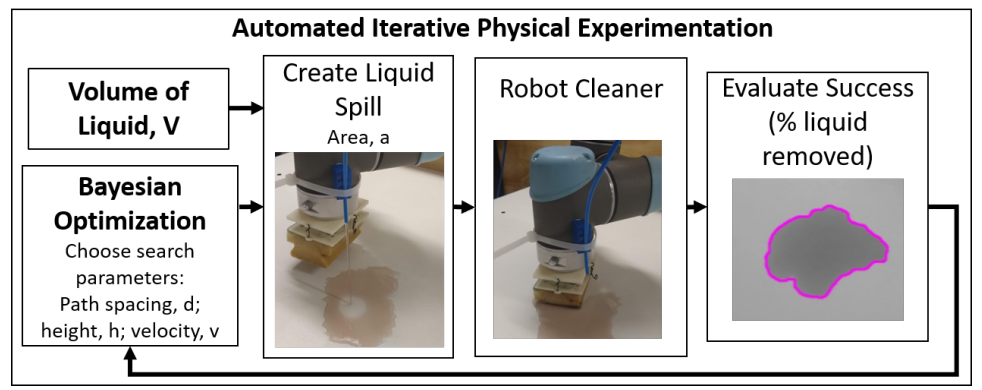

Fig. 1. Summary of the physical iterative experimentation approach to identify the optimal control parameters for cleaning a given volume of liquid.

Cleaning has been a long term goal for many research projects due to the challenge it poses for path planning, control strategies, manipulation, and sensing [8]. Autonomous cleaning robots have been fairly successful in structured environments when dealing with mess which can be modeled as solid particles [12]. In particular robot vacuum cleaners have been very successful and widely adopted [16]. However, many approaches are designed specifically for a single application like vacuuming or wiping, or for a specific cleaning tool [7]. Learning-based control strategies have been introduced as viable methods to solve the complexities involved with the problem of robotic cleaning by manipulation [10]. However, to make this a viable solution, the problem must be cleverly formulated to make the learning tractable, safe, and general purpose. Learning by imitation of humans has also been proposed for generating effective cleaning trajectories [9], but has limited generality. To move towards developing robots that can clean liquids,new approaches are required as the problem cannot be easily modeled. The liquid on a surface is complex and secondly the interaction between the liquid and the cleaning device (for example) a cleaning sponge is also very hard to model. Whilst there has been some examples of cleaning liquids, for example with window cleaning robots [11], there has been limited exploration of the optimal cleaning trajectories for liquids.

Thus, to develop cleaning algorithms and address this complex physical problem, we propose a new approach for developing control solutions for the cleaning of liquid spills. In this method, a robot has been developed which can physically iterate over the problem, and monitor its own success, allowing the system to learn and optimize the control parameters through physical experimentation of the cleaning progress. This approach is summarized in Fig. 1 showing that for a given volume of liquid the experimental process is fully automated. From this, we can extract and extrapolate rules for cleaning liquid spills of different sizes. This novel framework for developing the control approaches for cleaning liquid spills is fully automated allowing a single cleaning experiment to be performed in around 3 minutes. The learning and optimization process is shown to achieve control solutions which achieve performance that matches or exceeds human performance. In particular, in this paper we make the following contributions: 
- A base algorithm for liquid cleaning, with adjustable parameters

- An autonomous experimental framework for continuous exploration and optimization of liquid cleaning trajectory

- Experimental evidence demonstrating the validity of the system, and showing the ability to find controllers for different hardware solutions through iterative physical experimentation

This work presents an original framework for robotic liquid cleaning, with the methods given in Section II. Following this, details of the experimental setup are given. The experimental results are given in Section IV, followed by a discussion and conclusion on the wider role of this approach for the development of control strategies for physical tasks which are hard to model.

\section{Methods}

As shown in Fig. 1, physical experimentation is used to obtain the relevant control parameters, an approach which has previously been applied to other control problems $[15,14,3]$. A base cleaning algorithm is optimized using feedback which is extracted automatically using computer vision. This section presents this iterative method.

\subsection{Cleaning Algorithms}

A template, parameterized cleaning path has been devised which is then optimized through the iterative process. This parameterized path has been developed from human intuition and experience of performing the cleaning action. Parameters have been chosen which are believed to have the biggest influence on cleaning behaviors. The path is parameterized by three key variables. Firstly the space step, $d$, the distance between points on the edge of the liquid spill, measured parallel to the major axis. Secondly, $h$, the height by which the sponge is compressed. The final parameter, $v$, is the velocity of movement along the path. The cleaning path is centered around the major axis of the spill which is identified for every liquid spill. The robot end-effector is kept parallel to the long axis of the liquid spill.

Fig. 2 demonstrates these parameters with the range of values considered is given in Table 1. Thus, each cleaning attempt, $c_{i}$, can be described by the vector $[d, h, v]$. Human intuition is necessary to develop a base cleaning algorithm to make this a tractable problem. Although this does limit the search space, it still allows for a wide variety of different cleaning strategies to emerge. The control and parameters were chosen to be as generic as possible, however, there may need to be some adaptation for liquids with a significantly different viscosity or foaming properties.

\subsection{Optimization}

To identify the optimal cleaning parameters, $c$, for a given volume $V$ of liquid spill an optimization algorithm must be used. This task requires the optimization of a 
Table 1. Parameters of the cleaning algorithm showing the minimum and maximum values and also the parameters chosen for the general wipe.

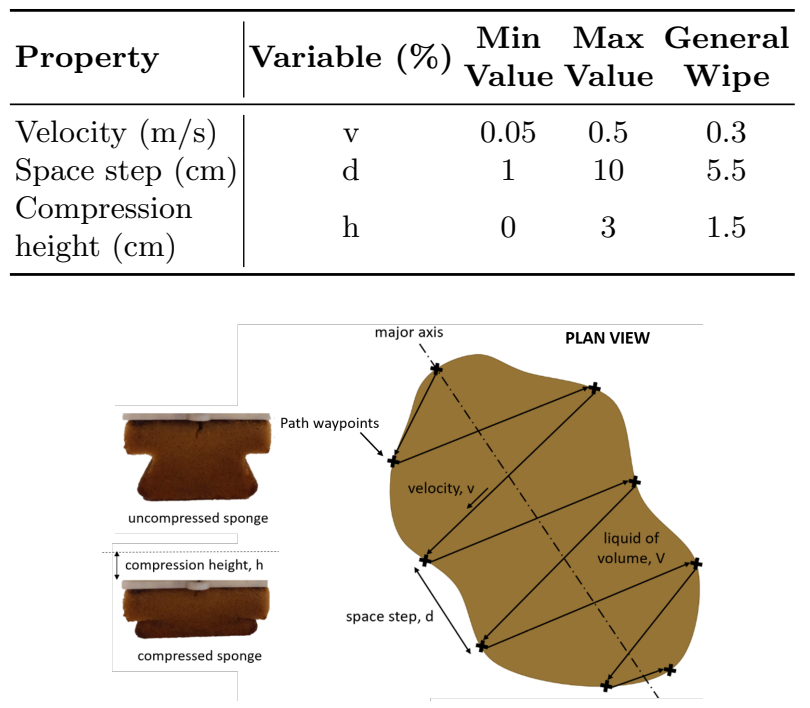

Fig. 2. Diagram of path planning and parameterization of the path. Inset showing how the compression height is defined.

complex dynamic system with environmental interactions which are challenging to models. Thus, an optimization algorithm that does not rely on an accurate physical model is needed [18]. Bayesian optimization is such a method and can sample efficiently from black-box functions without requiring gradient information. It is a method employed to minimize the number of iterations required to find the maximum of an objective function $f(\theta)$, where $\theta$ is the parameter vector. Thus the cleaning parameters to be next tested, $c_{n+1}$, are determined by the objective function, in this case the liquid removal performance, $P$ :

$$
c_{n+1}=\underset{c}{\operatorname{argmax}} P(c)
$$

We employ a typical Matern kernel (with smoothness parameter $\varsigma=2.5$ ) as defined in [13]. The exploration parameter $(\kappa)$ of the acquisition function has been tuned based on the Gaussian process Upper Confidence Bound with a value of $\kappa=80$ used to favor exploration. The optimization was implemented using the BayesianOptimization library for Python ${ }^{1}$. To ensure the optimization process explored the full area, the optimization was initialized by testing points where two parameters were held at the mid range and the other was at $25 \%$ or $75 \%$ of the range. Performance was evaluated by comparing the initial area of liquid on the surface to the area remaining after cleaning and taking the percentage reduction in area.

\footnotetext{
${ }^{1}$ https://github.com/fmfn/BayesianOptimization
} 

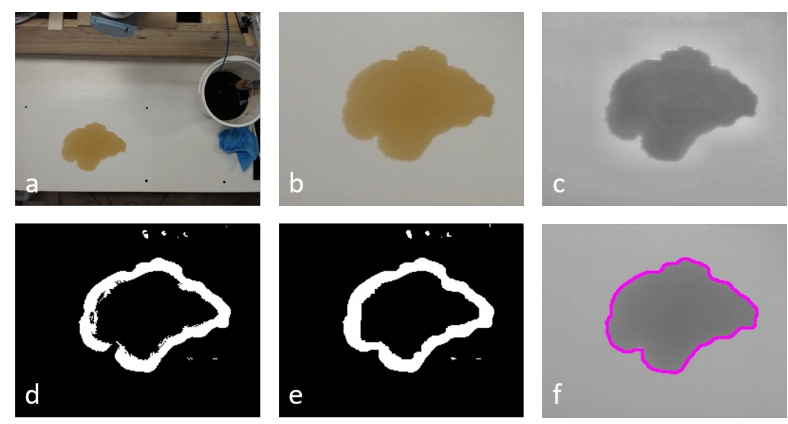

Fig. 3. Vision pipeline. (a) Original image, (b) cropped image, (c) enhancing contrast, (d) thresholding, (e) closing mask, (f) external contours selection.

\subsection{Visual Identification of Liquids}

This approach relies on the ability to correctly identify the location and area of the liquid spill, but visually identifying liquids is a challenging problem due to the reflection, refraction, and variability in presentation [5]. It is important that the area is estimated accurately as the area evaluation is used to form the acquisition function of the optimization process.

Fig. 3 shows the vision pipeline which has been developed to accurately identify the area and location; it has the following stages:

- Cropping: The image is cropped to a predetermined region of the surface.

- Contrast enhancement: Contrast-limited adaptive histogram equalization is applied to enhance the definition of the edge of the liquid.

- Thresholding: Adaptive mean thresholding with an inverted output is applied to account for varying lighting conditions.

- Mask closing: Erosion followed by dilation is applied to close the mask. This ensures a continuous contour running around the edge of the liquid.

- Contour selection: Only external contours are selected to account for the hollow nature of the mask and are converted to area. Contours smaller than 40 square pixels $\left(1.3 \mathrm{~cm}^{2}\right)$ are discarded as noise.

Weak coffee is used in the experiments as the base liquid. Using this pipeline it is possible to identify the area of liquid before cleaning, $a_{0}$, and the area after removal, $a_{r}$. Thus the performance for each cleaning process can be determined:

$$
p_{i}=\left(1-\frac{a_{r i}}{a_{0 i}}\right) \cdot 100 \%
$$

The accuracy of the vision system has been tested for 20 liquid spills by comparing that found with the pipeline to the ground truth, which was found using manual thresholding with the software Image J and a 30-centimeter ruler as a reference object [1]. The results (Fig. 4) show a high accuracy with approximately less than $1 \%$ error. 


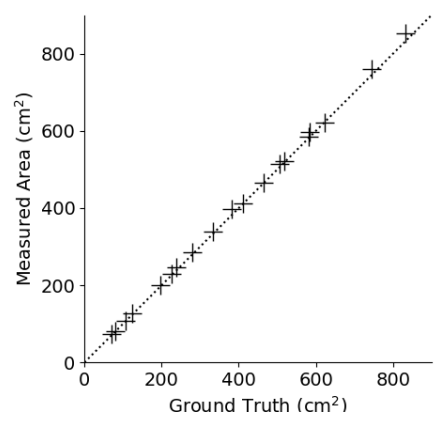

Fig. 4. Accuracy of the vision system, showing the estimated accuracy against a ground truth for a range of areas of liquid.

\section{$3 \quad$ Experimental Setup}

An experimental setup has been developed to automate the physical tests. This allows for many experiments to be carried out and for conditions to be kept constant, so successive experiments can be compared. The experimental setup is shown in Fig. 5, with the experimental process detailed in Fig. 5 . Mounted on the end-effector of a UR5 arm is a tube connected to a custom designed syringe pump, which has limit switches which determine the volume of liquid that will be dispensed. The pump is connected to a large liquid reservoir. After dispensing a given volume of liquid, the overhead camera is used to identify the area of liquid present on the kitchen work surface, $a_{0 i}$. Using the parameters chosen by the optimizer, the robot carries out the cleaning process using the sponge mounted on the end-effector.

After cleaning, the camera is used again to detect the amount of liquid remaining, $a_{r i}$, and hence evaluate the success of the cleaning process. To allow the experiment to be repeated, the setup must then be returned to the original state. To empty the sponge, a liquid removal system has been developed. The cleaning device (in this case a sponge) is pressed onto the central disc of the liquid removal setup, with the compression forcing liquid out of the material. The robot end-effector tilts forwards and backwards to remove more liquid. When liquid flows out of the material it drains through the mesh into the container beneath. The dry sponge is then used to perform a full grid-wise sweep of the surface to remove any liquid that may remain. The parameters were chosen manually for this general wipe process based on trial and error (see Table 1 for values). To ensure the surface was fully clean, this process was repeated twice. After wiping, the sponge is emptied and the next experiment could begin.

This setup has been shown to be highly reliable at delivering a liquid spill of a given volume and providing high reproducibility across tests. For a sequence of tests $(\mathrm{n}=12)$ where $28 \mathrm{ml}$ was deposited on the surface, the shape was consistently circular and the area of the liquid patch averaged $496.4 \mathrm{~cm}^{2}\left(\mathrm{~s}=12.9 \mathrm{~cm}^{2}\right)$. 

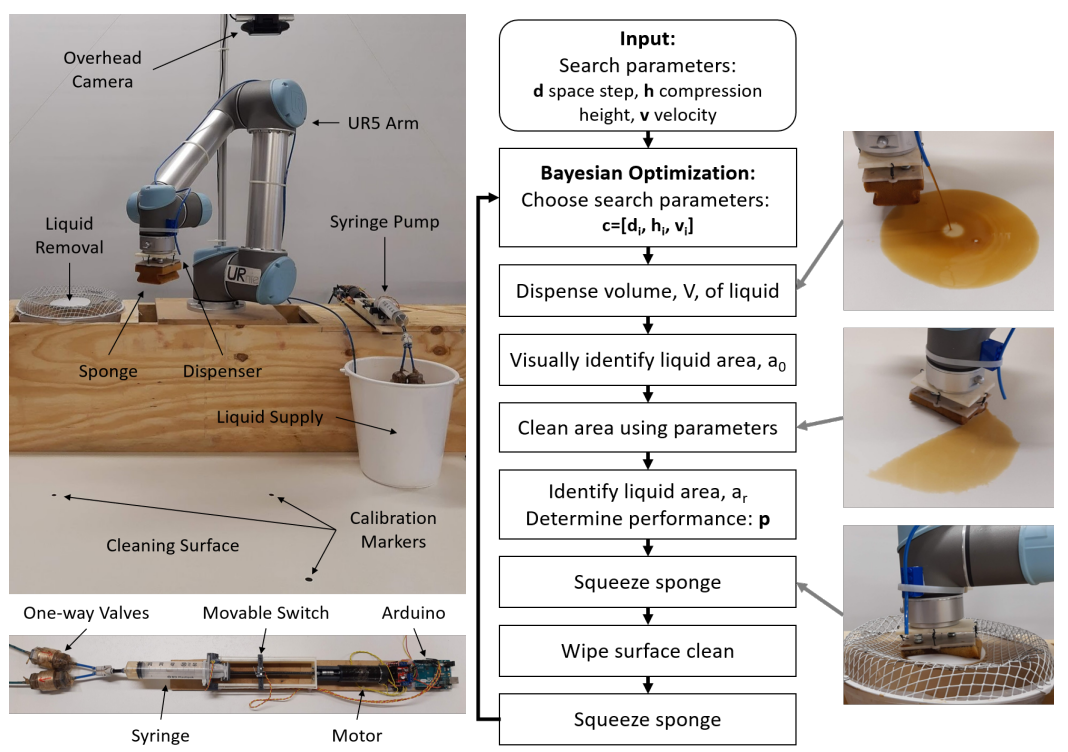

Fig. 5. Left) The experimental setup in a kitchen setup, showing the key parts: the syringe pump for dispensing liquid, the liquid removal system, sponge and liquid dispenser. Right) Flowchart of the full experimental procedure for obtaining optimal control parameters.

On average, it takes around 3 minutes for each experiment to be undertaken, allowing the system to rapidly test and iterate.

\section{Results}

\subsection{Optimization for a Fixed Volume of Liquid}

The results of the automated optimization of cleaning parameters for a fixed volume, in this case, $28 \mathrm{ml}$, are shown in Fig. 6 . To contextualize the performance of the system, the effectiveness of human cleaning and a naive algorithm was measured and have been added for comparison. The human was subject to only two constraints on cleaning: a maximum of 15 seconds to clean (the average time of the robot movement) and that they could not remove liquid from the sponge during cleaning. The naive baseline used a side to side wiping pattern with non-optimized parameters using the general clean parameters specified in Table 1, thus providing a non-optimized robot clean for comparison.

The results show that after 35 iterations (approximately 2 hours of experiments) the optimal parameters are found. The velocity settles the quickest, and thus successful cleaning at this volume is least sensitive to the velocity of the path. The compression height, $h$, is the last variable to be optimized, to a height of around $2 \mathrm{~cm}$. The spacing of the path optimizes at a similar speed to compression height with an optimal value of around $4 \mathrm{~cm}$. The performance appears to be sensitive to small changes in control parameter. 

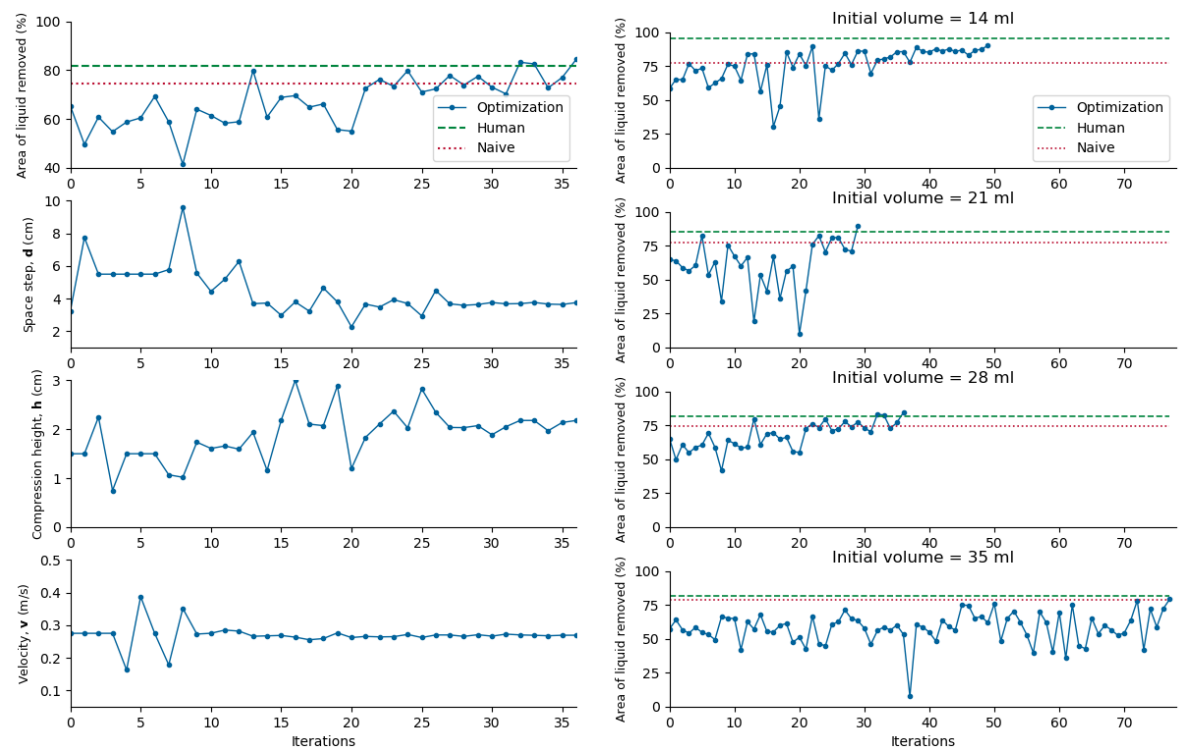

Fig. 6. Left)Bayesian optimization of the cleaning process for a liquid spill with a volume of $28 \mathrm{ml}$. Showing the removal success and the exploration of the parameters. Right) The performance of the optimization process for different volumes of liquid throughout the optimization process.

The optimal performance that is found shows the potential to exceed that of human performance. When cleaning, the human approaches this as an open loop process and could return to unsuccessfully cleaned patches whereas the robot is closed loop. To approach, and indeed exceed human performance shows the effectiveness of this approach. It shows that the optimization process finds specific control parameters with a degree of accuracy and precision in execution which a human would struggle to identify and perform.

\subsection{Optimal Control Parameters for Varying Volume Spills}

The automated optimization process has been run on three additional volumes of liquid to understand how the optimum control parameter varies for different volumes of liquid. Fig. 6 shows the performance throughout the optimization process for the four different volumes of liquid explored. Again, for each volume of liquid tested, the human success rate and that of a naive algorithm are presented for comparison. In general, the optimization process takes longer for the larger volumes of liquid. For the largest spill it took over 70 experiments, which equates to nearly 4 hours of experiments. In all cases the optimized performance exceeds or shows near to human-level performance. However, the robot system output performs humans better for larger volumes of liquid.

Fig. 7 compares the optimal control parameters for different size of liquid spills. For larger spills, a larger space step provides better performance with an 

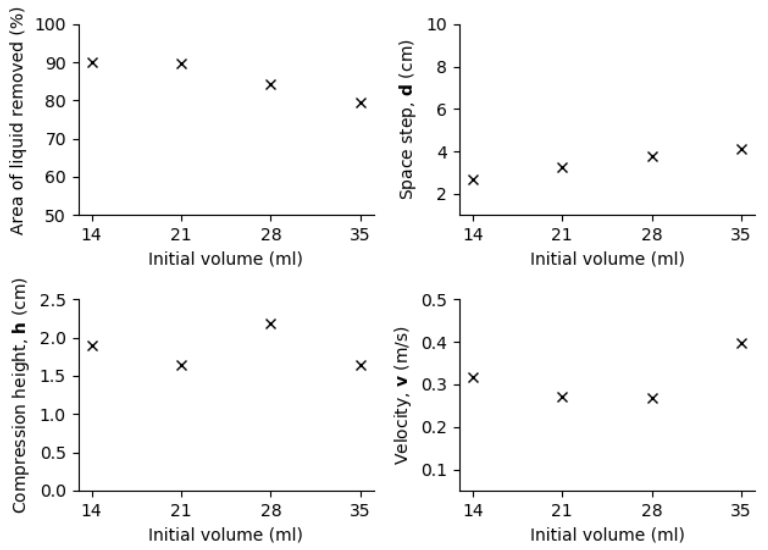

Fig. 7. The optimal parameters that were found for different volumes of liquid spill, and the overall performance that was achieved.

approximately linear relationship between liquid spill volume and space step. The effect of velocity is more complex; for larger spills a higher velocity is found to be optimal, but prior to this, decreasing velocity is found to improve performance. The optimal compression height is found to be similar across all volumes of liquid. In summary, these results show the complexity of the cleaning problem.

To test the sensitivity of the performance on the cleaning parameters on different volumes, the optimal parameters for a given volume, $c_{v, o p t}$, were tested on a range of other volumes of liquid spill. Fig. 8 shows the results; it can be observed that there is a significant decrease in performance when the optimal parameters are not used. Thus, using the optimal parameters for a given volume of liquid is necessary to achieve optimal performance. This validates the necessity of this process, and that the optimal parameters found for the different volumes provide the best performance despite the lack of obvious trends in the selection of the control parameters with respect to the volume of liquid.

\subsection{Parameter Generalization}

Using the optimal control parameters for different volumes of liquid, parameters can be predicted for other volumes using regression. Linear regression was used for the spacing size of the path, compression height is assumed to be a constant, and cubic regression is used for the velocity of the sponge. Fig. 9 shows the cleaning performance when parameters are chosen using regression in comparison to those chosen through the optimization process. These are also compared to cleaning using the parameters found for $35 \mathrm{ml}$.

Using this regression based approach to predict the optimal in most case, exceeds human performance. The predicted parameters consistently out perform the naive algorithm and that using the $35 \mathrm{ml}$ parameters. In many cases, in 

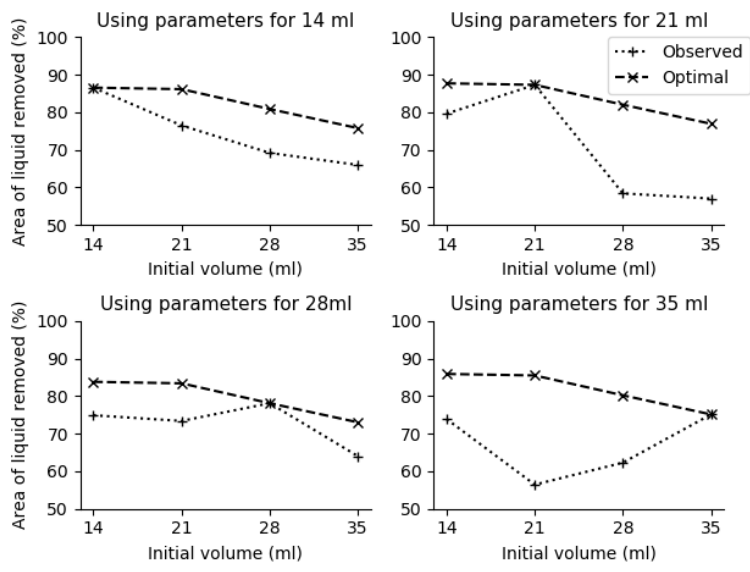

Fig. 8. The optimal parameters for a given volume of liquid and applied to other volumes of liquid to test the sensitivity.

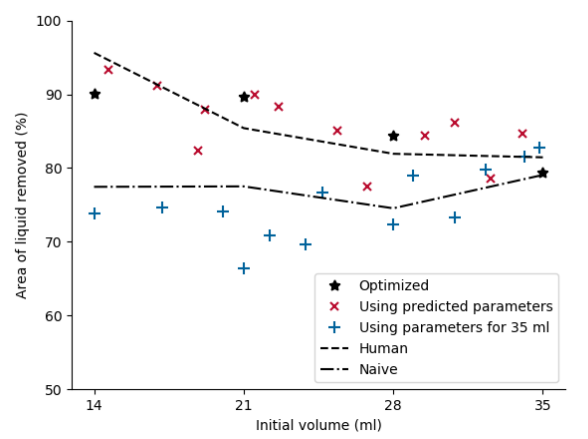

Fig. 9. Performance of the parameters predicted by regression for previously untested volumes in comparison to the $35 \mathrm{ml}$ parameters.

particular around the middle of the range of volumes tested, the performance exceeded the human baseline considerably.

\subsection{Comparison of Different Cleaning Methods}

This approach can be shown to generalize to other cleaning approaches. The cleaning process was also optimized for a J-cloth. Fig. 10 shows a comparison of the optimization for a J-cloth and the sponge for a $28 \mathrm{ml}$ liquid spill. The J-cloth performance was found to be more sensitive to the input parameters, especially the compression height (Fig. 10). However, the optimal performance is higher than that of the sponge. This experiment demonstrates how this setup could be used to allow comparison between different cleaning techniques, and to enable the optimal technique to be found for a given volume of liquid. 


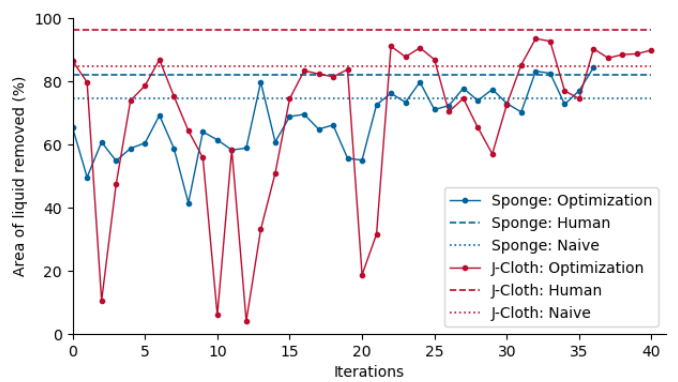

Fig. 10. Optimization of two different cleaning materials (sponge and J-cloth) for a liquid spill with a volume of $28 \mathrm{ml}$.

\section{Conclusions}

This approach of iterative finding the optimal control parameters for the complex physical process of cleaning liquids has been shown to find parameters which can in some cases outperform humans. By automating the process it has been possible to carry out over 400 experiments to allow the optimal parameters to be found for given volumes of liquid. The use of regression to generalize the results found for a wider range of volumes was shown to work well, and enable this approach to be practically useful on variety of different sized liquid spills. This work focused on cleaning water-based liquid spills as it is both a widely occurring liquid spill type and challenging cleaning tasks due to the low viscosity of water. In future work, other liquids with varying properties should be investigated, alongside different cleaning materials.

This approach of using robotics to automate the finding of optimal control parameters has been shown to effective. Within the cleaning domain this could be expanded to optimizing for different cleaning devices or for more complex cleaning scenarios. It is also more widely applicable in scenarios which are challenging to model by developing continuous robotic exploration approaches.

\section{Acknowledgment}

This work was supported by BEKO PLC and Symphony Kitchens. We are especially thankful for the valuable inputs from Dr Graham Anderson and Dr Natasha Conway.

\section{References}

1. Abràmoff, M.D., Magalhães, P.J., Ram, S.J.: Image processing with imagej. Biophotonics international 11(7), 36-42 (2004)

2. Alonso, I.G., Fernández, M., Maestre, J.M., Fuente, M.d.P.A.G.: Service robotics within the digital home: applications and future prospects, vol. 53. Springer Science \& Business Media (2011)

3. Brodbeck, L., Hauser, S., Iida, F.: Morphological evolution of physical robots through model-free phenotype development. PloS one 10(6), e0128444 (2015) 
4. Bugmann, G., Copleston, S.N.: What can a personal robot do for you? In: Conference Towards Autonomous Robotic Systems. pp. 360-371. Springer (2011)

5. Eppel, S., Kachman, T.: Computer vision-based recognition of liquid surfaces and phase boundaries in transparent vessels, with emphasis on chemistry applications. arXiv preprint arXiv:1404.7174 (2014)

6. Flores, G.E., Bates, S.T., Caporaso, J.G., Lauber, C.L., Leff, J.W., Knight, R., Fierer, N.: Diversity, distribution and sources of bacteria in residential kitchens. Environmental microbiology 15(2), 588-596 (2013)

7. Kabir, A.M., Langsfeld, J.D., Shriyam, S., Rachakonda, V.S., Zhuang, C., Kaipa, K.N., Marvel, J., Gupta, S.K.: Planning algorithms for multi-setup multi-pass robotic cleaning with oscillatory moving tools. In: 2016 IEEE International Conference on Automation Science and Engineering (CASE). pp. 751-757. IEEE (2016)

8. Kemp, C.C., Edsinger, A., Torres-Jara, E.: Challenges for robot manipulation in human environments [grand challenges of robotics]. IEEE Robotics \& Automation Magazine 14(1), 20-29 (2007)

9. Kim, H., Lee, H., Chung, S., Kim, C.: User-centered approach to path planning of cleaning robots: analyzing user's cleaning behavior. In: Proceedings of the ACM/IEEE international conference on Human-robot interaction. pp. 373$380(2007)$

10. Kim, J., Mishra, A.K., Limosani, R., Scafuro, M., Cauli, N., Santos-Victor, J., Mazzolai, B., Cavallo, F.: Control strategies for cleaning robots in domestic applications: A comprehensive review. International Journal of Advanced Robotic Systems 16(4), 1729881419857432 (2019)

11. Miyake, T., Ishihara, H.: Mechanisms and basic properties of window cleaning robot. In: Proceedings 2003 IEEE/ASME International Conference on Advanced Intelligent Mechatronics (AIM 2003). vol. 2, pp. 1372-1377. IEEE (2003)

12. Prassler, E., Ritter, A., Schaeffer, C., Fiorini, P.: A short history of cleaning robots. Autonomous Robots 9(3), 211-226 (2000)

13. Rasmussen, C.E.: Gaussian processes in machine learning. In: Summer School on Machine Learning. pp. 63-71. Springer (2003)

14. Rosendo, A., von Atzigen, M., Iida, F.: The trade-off between morphology and control in the co-optimized design of robots. PloS one 12(10), e0186107 (2017)

15. Saar, K.A., Giardina, F., Iida, F.: Model-free design optimization of a hopping robot and its comparison with a human designer. IEEE Robotics and Automation Letters 3(2), 1245-1251 (2018)

16. Sahin, H., Guvenc, L.: Household robotics: autonomous devices for vacuuming and lawn mowing [applications of control]. IEEE Control Systems Magazine 27(2), 20$96(2007)$

17. Samseth, J., Banford, A., Batandjieva-Metcalf, B., Cantone, M.C., Lietava, P., Peimani, H., Szilagyi, A.: Closing and decommissioning nuclear power reactors. UNEP Year book pp. 35-49 (2012)

18. Snoek, J., Larochelle, H., Adams, R.P.: Practical bayesian optimization of machine learning algorithms. In: Advances in neural information processing systems. pp. 2951-2959 (2012)

19. Van Rensbergen, G., Pacolet, J.: Instrumental activities of daily living (i-adl) trigger an urgent request for nursing home admission. Archives of public health $=$ Archives belges de sante publique $\mathbf{7 0}(1), 2$ (2012)

20. Zachiotis, G.A., Andrikopoulos, G., Gornez, R., Nakamura, K., Nikolakopoulos, G.: A survey on the application trends of home service robotics. In: 2018 IEEE International Conference on Robotics and Biomimetics (ROBIO). pp. 1999-2006. IEEE (2018) 